\title{
AN INITIAL VALUE PROBLEM FOR HYPERBOLIC DIFFERENTIAL EQUATIONS
}

BENHAM M. INGERSOLL

We consider the normal form of the linear partial differential equation of hyperbolic type

$$
L[u] \equiv u_{x y}+a u_{x}+b u_{y}+c u=d .
$$

It is well known that if the coefficients of (1) satisfy certain continuity conditions, a unique solution $U(x, y)$ of $(1)$ can be determined over any rectangle with sides parallel to the coordinate axes which lies entirely within the domain of continuity of the coefficients of (1) by prescribing the solution along any two adjacent sides of the rectangle. ${ }^{1}$ No generality will be lost by assuming that the adjacent segments lie on the coordinate axes, so that a vertex of the rectangle is on the origin.

It will be shown here that for a certain sub-class of equations of this type a unique solution is obtainable by prescribing merely two partial derivatives of $u(x, y)$, one along each of two adjacent sides of the rectangle, that is, by prescribing $\partial^{k} u(x, y) /\left.\partial x^{k}\right|_{x=0}$ and $\partial^{m} u(x, y) /\left.\partial y^{m}\right|_{y=0}$ where $k$ and $m$ are any non-negative integers, instead of $u(x, 0)$ and $u(0, y)$. This result is obtained by reducing the new problem to the classic one $(k=m=0)$.

It should also be noted that the result to be proved complements, in a certain sense, results of Bergman ${ }^{2}$ on elliptic differential equations. If $a=\sum_{m, n} a_{m n} x^{m} y^{n}, b=\bar{a}, c=\sum_{m, n} c_{m n} x^{m} y^{n}, c_{m n}=c_{n m}$, are entire functions of two complex variables $x$ and $y$ where $y$ is conjugate to $x$, and we write $x=X+i Y, y=X-i Y$; then (1) becomes an equation of elliptic type in the $X, Y$ plane. It has been shown, by means of Bergman's operator method, that in order to determine the regularity domain of a solution $U=\sum_{m, n} U_{m n} x^{m} y^{n}, U_{m n}=\bar{U}_{n m}$, from a given subsequence $\left\{U_{m k}\right\}, k$ fixed, $m=0,1,2, \cdots$, it is sufficient to know the subsequences $\left\{a_{m \nu}\right\},\left\{a_{\nu m}\right\},\left\{c_{m \nu}\right\}, \nu=0,1,2, \cdots, k, m=0,1,2$, $3, \cdots$, of the coefficients in the power series expansion of $a$ and $c$ in (1). If, by the same change of variables, the result of the present paper is formulated as a theorem for equations of elliptic type, it becomes

Presented to the Society, October 28, 1944, under the title On singularities of solutions of partial differential equations. III; received by the editors July 12, 1945, and, in revised form, January 19, 1948.

${ }^{1}$ See Courant and Hilbert, Mathematische Physik, vol. 2, pp. 315-316.

${ }^{2}$ See S. Bergman, Linear operators in the theory of partial differential eqnations, Trans. Amer. Math. Soc. vol. 53 (1945) pp. 130-155, in particular p. 141. 
apparent that our results belong to somewhat the same range of ideas.

We first treat a simple example which will indicate the method of proof in the general case.

We wish to determine a solution $u(x, y)$ of the partial differential equation

$$
u_{x y}+u_{x}+u_{y}+u=0
$$

which satisfies the initial conditions

$$
u_{y y}(x, 0)=x, \quad u_{x x}(0, y)=y .
$$

Differentiating (A) with respect to $y$, then setting $y=0$ and employing the first of the conditions (B), there results the system of ordinary linear differential equations for the functions $u(x, 0)$, $u_{y}(x, 0)$

$$
\begin{aligned}
u_{y}^{\prime}(x, 0)+u^{\prime}(x, 0)+u_{y}(x, 0)+u(x, 0) & =0, \\
u_{y}^{\prime}(x, 0)+u_{y}(x, 0) & =-x-1,
\end{aligned}
$$

where primes denote derivatives with respect to $x$.

The complete integral of the system (C) is given by

$$
u(x, 0)=K_{1} e^{-x}+x, \quad u_{y}(x, 0)=K_{2} e^{-x}-x .
$$

If each equation of the system (C) is differentiated once, upon adjoining to $(C)$ the new equations and setting $x=0$ in the resulting system, we obtain by employing the initial conditions (B),

$$
\begin{aligned}
u_{y}^{\prime}(0,0)+u^{\prime}(0,0)+u_{y}(0,0)+u(0,0) & =0, \\
u_{y}^{\prime}(0,0)+u_{y}(0,0) & =-1, \\
u_{y}^{\prime}(0,0)+u^{\prime}(0,0) & =-1, \\
u_{y}^{\prime}(0,0) & =-2 .
\end{aligned}
$$

Hence $u_{y}(0,0)=1, u(0,0)=0$; thus $K_{1}=0, K_{2}=1$, and $u(x, 0)=x$, $u_{y}(x, 0)=e^{-x}-x$.

By symmetry, $u(0, y)=y, u_{x}(0, y)=e^{-y}-y$.

The original problem has now been reduced to one of the classical type, namely, the determination of a solution $u(x, y)$ of (A) satisfying the boundary conditions

$$
u(x, 0)=x, \quad u(0, y)=y .
$$

Riemann's integration method yields

$$
u(x, y)=0+\int_{0}^{y} e^{\eta-x-y}(1+\eta) d \eta+\int_{0}^{x} e^{\xi-x-y}(1+\xi) d \xi,
$$


that is, $u(x, y)=y e^{-x}+x e^{-y}$.

The following work follows the above lines.

We shall use the notation

$$
\frac{d^{i} f(x)}{d x^{i}}=f_{i}(x) ; \quad \frac{\partial^{i+j} \mathcal{u}(x, y)}{\partial x^{i} \partial y^{j}}=u_{i j}(x, y) ; \quad \frac{\partial^{i+j} L[u]}{\partial x^{i} \partial y^{j}}=L_{i j}(u) .
$$

By $\alpha_{i j ; \xi \beta} \equiv \alpha_{i j ; \xi \beta}(x, y)$ we shall mean the coefficient of $u_{\xi \beta}(x, y)$ in $L_{i j}[u]$. The letters $k$ and $m$ will be thought of as fixed integers throughout the paper. If in an equation containing $x$ and $y$ such as (1), we wish, for example, to change $(x, y)$ to $(3,4)$, we shall refer to it as the equation $(1 ; 3,4)$.

Form the system of equations

$$
L_{i j}[u]=d_{i j} \quad(i=0, \cdots, k-1 ; j=0, \cdots, m-1)
$$

and the matrix of the $u_{\xi \beta}$ occurring in (2)

$$
\left(\begin{array}{ccc}
u_{00} & u_{01} \cdots \cdot u_{0 m} \\
u_{10} & u_{11} \cdots \cdot u_{1 m} \\
\cdot & \cdot \cdot \cdot \cdot \cdot \\
u_{k 0} & u_{k 1} \cdots \cdot u_{k m}
\end{array}\right)
$$

In the equations (2) when the terms containing $u_{k \beta}$ and $u_{\xi m}$ $(\beta=0, \cdots, m ; \xi=0, \cdots, k)$ are transposed to the right-hand side, there results a system of $k m$ equations in $k m$ functions $u_{i j}(i=0, \cdots$, $k-1 ; j=0, \cdots, m-1)$. We shall refer to these as the $(2)^{*}$ equations. Since the reordering of a system of equations cannot affect the absolute value of their determinant, we shall call

$$
A(x, y)
$$

the absolute value of the determinant of the system $(2)^{*}$ in the variables $u_{i j}, \quad(i=0, \cdots, k-1 ; j=0, \cdots, m-1)$. The elements of $A(x, y)$ are $a, b, c$ and their derivatives.

The subsets $L_{0 j}[u]=d_{0 j} \quad(j=0, \cdots, m-1)$ and $L_{i 0}[u]=d_{i 0}$ $(i=0, \cdots, k-1)$ of the system $(2)^{*}$ are fundamental in this paper, and we write them in the forms

$$
\begin{aligned}
\sum_{t} \alpha_{0 j ; 1 t} u_{1 t}+ & \sum_{t} \alpha_{0 j ; 0 t} u_{0 t}=d_{0 j} \\
& (0 \leqq j \leqq m-2 ; t=0, \cdots, j+1) \\
=d_{0 j}- & u_{1 m}-b u_{0 m} \\
& (j=m-1 ; t=0, \cdots, m-1),
\end{aligned}
$$




$$
\begin{aligned}
\sum_{\xi} \alpha_{i 0 ; \xi 1} u_{\xi 1}+\sum_{\xi} \alpha_{i 0 ; \xi 0} u_{\xi 0}=d_{i 0} & \\
(0 \leqq & (i \leqq k-2 ; \xi=0, \cdots, i+1) \\
=d_{i 0}-u_{k 1}-a u_{k 0} & (i=k-1 ; \xi=0, \cdots, k-1) .
\end{aligned}
$$

The determinant $\left|\alpha_{0 j ; 1 t}\right|$ of the terms $u_{1 t}$ in (5) will be denoted by

$$
B(x, y)=\left|\alpha_{0 ; ; 1 t}\right| \quad(j, t=0, \cdots, m-1),
$$

and the corresponding determinant of (6) by

$$
C(x, y)=\left|\alpha_{i 0 ; ! 1}\right|
$$$$
(i, \xi=0, \cdots, k-1) .
$$

We may now state our principal result.

Theorem 1. In the equation $L[u]=d$ let $a, b, c, d$, be of class $C^{k}$ in $x$ and $C^{m}$ in $y$ and let $f(x), g(y)$ be preassigned functions of classes $C^{k+1}$ and $C^{m+1}$ respectively, with $f_{k}(0)=g_{m}(0)$. Let

$$
\begin{array}{ll}
A(0,0) \neq 0, & \\
B(x, 0) \neq 0 & \left(0 \leqq x<s_{1}\right), \\
C(0, y) \neq 0 & \left(0 \leqq y<s_{2}\right) .
\end{array}
$$

Under these conditions there exists a unique solution $u(x, y)$ of $L[u]=d$ $\left(0 \leqq x \leqq s_{1}, 0 \leqq y<s_{2}\right)$, such that

$$
\begin{aligned}
& u_{0 m}(x, 0)=f(x) \\
& \left(0 \leqq x<s_{1}\right) \text {, } \\
& u_{k 0}(0, y)=g(y) \\
& \left(0 \leqq y<s_{2}\right) \text {. }
\end{aligned}
$$

The following proof holds for $m k \neq 0$. The slight changes necessary for the case when $m$ or $k$ is zero will be indicated.

We shall first prove a series of lemmas. The first three of the lemmas will show that all the terms in the matrix $(3 ; 0,0)$ are determined by the assignment of its first row and last column, or its first column and first row, or its last row and last column.

Lemma 1. Under the conditions of Theorem 1 the assignment of the values of the first row and last column of $(3 ; 0,0)$, namely

$$
\begin{array}{lrl}
u_{0 j}(0,0) & =u_{0 j}^{*} & (j=0, \cdots, m-1), \\
u_{i m}(0,0) & =f_{i}(0) & (i=0, \cdots, k)
\end{array}
$$

together with the equations $(2 ; 0,0)$ uniquely determine all the terms in the matrix $(3 ; 0,0)$.

In using the values $(14)$ in $(5 ; 0,0)$, we note that only the terms 
in $u_{1 t}(0,0)(t=0, \cdots, m-1)$ remain to be found, but since $B(0,0)$ $\neq 0$, we can solve uniquely for these values. On differentiating the equations (5) once with respect to $x$, the only way terms in $u_{2 t}(x, y)$ $(t=0, \cdots, m-1)$ can occur is from differentiating the $u_{1 t}$ in the terms $\alpha_{0 j ; 1 t} u_{1 t}$. This shows that the determinant $\left|\alpha_{1 j ; 2 t}\right|=B(x, y)$. Hence we may find the $u_{2 t}(0,0)(t=0, \cdots, m-1)$ after having determined the $u_{1 t}(0,0)$. Owing to our continuity conditions on $a, b, c, d$, we may differentiate (5) at least $k-1$ times with respect to $x$. Hence the lemma is seen to hold.

Using the equations (6) as we did the equations (5), we may prove the following lemma.

Lemma 2. Under the conditions of Theorem 1 the assignment of the first column and last row of $(3 ; 0,0)$, namely

$$
\begin{array}{lrl}
u_{i 0}(0,0) & =u_{i 0}^{*} & (i=0, \cdots, k-1), \\
u_{k j}(0,0) & =g_{j}(0) & (j=0, \cdots, m)
\end{array}
$$

together with the equations $(2 ; 0,0)$ uniquely determine all the terms in the matrix $(3 ; 0,0)$.

LEMMA 3. If the conditions of Theorem 1 hold the assignment of values to the last row and last column

$$
\begin{aligned}
& u_{k j}(0,0)=g_{j}(0) \quad(j=0, \cdots, m-1), \\
& u_{i m}(0,0)=f_{i}(0) \quad(i=0, \cdots, k-1), \\
& u_{k m}(0,0)=f_{k}(0)=g_{m}(0)
\end{aligned}
$$

together with the system $(2 ; 0,0)$ uniquely determine all members of the matrix $(3 ; 0,0)$.

This follows from the facts that (16) determine all the right members of the system $(2 ; 0,0) *$ and the absolute value $A(0,0)$ of the determinant of this system of algebraic equations is not zero.

Since the functions $f(x)$ and $g(y)$ are assumed to be known, we may assign the values in (16); the resulting values to the elements of the matrix $(3 ; 0,0)$ will be denoted by

$$
u_{i j}(0,0)=F_{i j}(f, g) \text {. }
$$

In the equations $(5 ; x, 0)$ write

$$
\begin{array}{ll}
u_{0 i}(x, 0)=z^{i}(x), & u_{1 i}(x, 0)=\frac{d}{d x} z^{i}(x) \quad(0 \leqq i \leqq m-1), \\
u_{0 m}(x, 0)=f(x), & u_{1 m}(x, 0)=f_{1}(x) .
\end{array}
$$


This gives us the system of ordinary differential equations

$$
\begin{aligned}
\sum_{t} \alpha_{0 j ; 1 t}(x, 0) \frac{d z^{t}}{d x}+\sum_{t} \alpha_{0 j ; 0 t}(x, 0) z^{t}=d_{0 j}(x, 0) \\
(0 \leqq j \leqq m-2 ; t=0, \cdots, j+1) \\
=d_{0 j}(x, 0)-f_{1}(x)-b(x, 0) f(x) \\
(j=m-1 ; t=0, \cdots, m-1) .
\end{aligned}
$$

We assign as initial values to $z^{0}, \cdots, z^{m-1}$,

$$
z^{i}(0)=F_{0 i}(f, g) \quad(i=0, \cdots, m-1) .
$$

Since $\left|\alpha_{0 j ; 1 t}(x, 0)\right|=B(x, 0) \neq 0$ for $0 \leqq x<s_{1}$, and because of the continuity conditions satisfied by the coefficients of (1), the system $\left(5^{\prime}\right)$ is seen to have a unique solution ${ }^{3}$ of class $C^{k}$. Since the system $(2 ; 0,0)$ can be formed as follows

$$
\left\{\frac{d}{d x^{i}}\left[\left.L_{0 j}(u)\right|_{y=0}\right]\right\}_{x=0}=\left\{\frac{d}{d x^{i}}\left[\left.d_{0 j}(x, y)\right|_{y=0}\right]\right\}_{x=0},
$$

we see on adding to the system $\left(5^{\prime}\right)$ all equations obtained by differentiating it $1,2, \cdots, k-1$ times with respect to $x$, and then putting $x=0$, we have the system $(2 ; 0,0)$. In this system $\left(18^{\prime}\right)$ assures

$$
u_{i m}(0,0)=f_{i}(0) \quad(i=0, \cdots, k) .
$$

Thus from (17), (19), (20), and Lemma 1 we have:

LEMma 4. Under the conditions of Theorem 1 the unique solution of equations $\left(5^{\prime}\right)$ with initial conditions (19) satisfies the conditions

$$
\left.\frac{d^{i} z^{i}(x)}{d x^{i}}\right|_{x=0}=F_{i j}(f, g) \quad(i=0, \cdots, k ; j=0, \cdots, m-1) .
$$

In the equations $(6 ; 0, y)$ we make the replacements

$$
\begin{array}{ll}
u_{i 0}(0, y)=w^{i}(y), & u_{i 1}(0, y)=\frac{d w^{i}}{d y} \quad(0 \leqq i \leqq k-1), \\
u_{k 0}(0, y)=g(y), & u_{k 1}(0, y)=g_{1}(y) .
\end{array}
$$

Calling the resulting system of ordinary differential equations $\left(6^{\prime}\right)$, we assign initial values

$$
w^{i}(0)=F_{i 0}(f, g) \quad(i=0, \cdots, k-1) .
$$

We may treat $\left(6^{\prime}\right)$ as we treated $\left(5^{\prime}\right)$ and get the lemma.

\footnotetext{
${ }^{3}$ See E. L. Ince, Ordinary differential equations, p. 72.
} 
LEMma 5. Assuming conditions of Theorem 1 hold, the unique solution of system (6') with initial conditions (23) satisfies the conditions

$$
\left.\frac{d^{i} w^{i}(y)}{d y}\right|_{y=0}=F_{j i}(f, g) \quad(i=0, \cdots, m ; j=0, \cdots, k-1) .
$$

We now return to equation (1) and solve it by the classical theory for the unique solution with initial conditions

$$
\begin{aligned}
& u(x, 0)=z^{0}(x), \\
& 0 \leqq x<s_{1}, \\
& u(0, y)=w^{0}(y), \\
& 0 \leqq y<s_{2} \text {, }
\end{aligned}
$$

The solution of $L[u]=d$ for $0 \leqq x<s_{1}, 0 \leqq y<s_{2}$ so obtained we shall denote by $U(x, y)$. Theorem 1 will be complete if we can show

$$
U_{0 m}(x, 0)=f(x), \quad U_{k 0}(0, y)=g(y),
$$

and $U$ is the only such solution of $L[u]=d$.

$U(x, y)$ is unique, for Lemma 3 implies that $f(x)$ and $g(y)$, where $f_{k}(0)=g_{m}(0)$, together with (1) determine uniquely $(3: 0,0)$. Accordingly the other lemmas guarantee the uniqueness of the $z^{i}(x)$ and $w^{i}(y)(j=0, \cdots, m-1 ; i=0, \cdots, k-1)$, in particular that of $z^{0}(x)$ and $w^{\circ}(y)$, and thus that of $U$.

If from the equations $\left(5^{\prime}\right)$, say, we wish to consider a single one, for example, the one obtained by setting $j=7$, we shall designate it by $\left(5^{\prime}\right)(j=7)$, and shall use corresponding notation for other equations picked from sets.

In equations $\left(5^{\prime}\right)$ make the replacements

$$
\begin{array}{ll}
z^{j+1}=p^{j+1}, & \frac{d z^{j+1}}{d x}=\frac{d p^{j+1}}{d x} \\
f(x)=p^{m}, & f_{1}(x)=\frac{d p^{m}}{d x} .
\end{array}
$$

This yields $m$ differential equations

$$
\begin{array}{r}
\alpha_{0 j i 1, j+1}(x, 0) \frac{d p^{j+1}}{d x}+\alpha_{0 j ; 0, j+1}(x, 0) p^{j+1} \\
=d_{0 j}(x, 0)-\sum_{t} \alpha_{0 j ; 1 t}(x, 0) \frac{d z^{t}(x)}{d x}-\sum_{t} \alpha_{0 j ; 0 t}(x, 0) z^{t}(x) \\
(0 \leqq j \leqq m-1 ; t=0, \cdots, j)
\end{array}
$$

$\left[\left(5^{\prime \prime}\right)(j=m-1)\right.$ need not be written separately since $\alpha_{0 j ; 1, j+1}(x, 0)=1$ and $\left.\alpha_{0 j ; 0, j+1}(x, 0)=b(x, 0)\right]$. 
Now by $(5 ; x, 0)(j=0)$ and $(25), U_{01}(x, 0)$ is a solution of $\left(5^{\prime \prime}\right)$ $(j=0)$. By $\left(5^{\prime}\right)(j=0), z^{1}(x)$ is also a solution of $\left(5^{\prime \prime}\right)(j=0)$. (26) and Lemma 5 imply that $U_{01}(x, 0)=d w^{0}(y) /\left.d y\right|_{y=0}=F_{01}(f, g)$. But by $(19), z^{1}(0)=F_{01}(f, g)$. Hence $U_{01}(x, 0)=z^{1}(x)$. If we use in natural order the remaining equations of the set $\left(5^{\prime \prime}\right)$, a similar argument will establish that $U_{0 j}(x, 0)=z^{j}(x), j=1, \cdots, m-1$, the only difference in the discussion being that the identities $U_{0 h}(x, 0)=z^{h}(x)$, $h=1, \cdots, j-1$, must be used together with $(25) .\left(5^{\prime}\right) \quad(j=m-1)$ shows that $f(x)$ is a solution of $\left(5^{\prime \prime}\right)(j=m-1)$, and $(5 ; x, 0)(j=m-1)$ together with $U_{0 j}(x, 0)=z^{j}(x), j=0, \cdots, m-1$, show that $U_{0 m}(x, 0)$ is also a solution. However, from $(26), U_{0 m}(0,0)=d^{m} w^{0}(y) /\left.d y^{m}\right|_{y=0}$. But by prescription, that is, by $\left(18^{\prime}\right), f(0)=F_{0 m}(f, g)$, and hence, by Lemma $5, f(0)=d^{m} w^{0}(y) /\left.d y^{m}\right|_{y=0}$. Therefore $U_{0 m}(x, 0)=f(x)$. It can be shown in a similar way that $U_{k 0}(0, y)=g(y)$.

The slight change necessary in the case $m k=0$ is as follows. Say, for example, that $k=0$. An entity arising in much the same way and playing the same role as an entity $(n)$ in the previous work will be denoted by $(\tilde{n})$.

Form the system

$$
L_{0 j}[u]=d_{0 j} \quad(j=0, \cdots, m-1)
$$

and the matrix of the $u_{\xi \beta}$ occurring in ( $\left.\tilde{2}\right)$

$$
\left(\begin{array}{lll}
u_{00} & u_{01} \cdots u_{0 m} \\
u_{10} & u_{11} \cdots u_{1 m}
\end{array}\right)
$$

$(\tilde{2})^{*}$ will be formed by transposing to the right-hand side in the system

( $\tilde{2})$ those terms containing $u_{1 m}$ and $u_{0 m}$. $(\widetilde{5})$ will then be the same as

(5). The $z^{i}(x), i=0, \cdots, m-1$, will be obtainable as before, $(\tilde{5})$ playing the roles of both (2)* and (5). Lemmas 2,3 , and 5 will not be used. Solving (1) by the classical theory for the unique solution with initial conditions

$$
\begin{aligned}
& u(x, 0)=z^{0}(x), \\
& u(0, y)=g(y),
\end{aligned}
$$

the rest of the proof will go through as before with Lemma 1 leading to uniqueness and $g(y)$ taking the place of $w^{0}(y)$.

Crty College of New York 